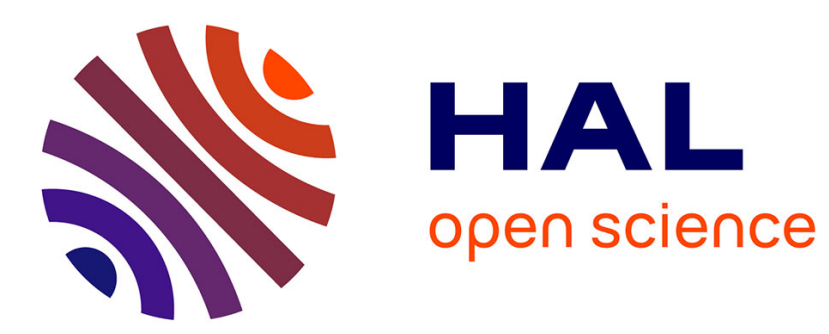

\title{
Thermal transfer in amorphous superionic Li2S
}

Yanguang Zhou, Sebastian Volz

\section{To cite this version:}

Yanguang Zhou, Sebastian Volz. Thermal transfer in amorphous superionic Li2S. Physical Review B, 2021, 103 (22), pp.224204. 10.1103/physrevb.103.224204 . hal-03420114

\section{HAL Id: hal-03420114 https://hal.science/hal-03420114}

Submitted on 9 Nov 2021

HAL is a multi-disciplinary open access archive for the deposit and dissemination of scientific research documents, whether they are published or not. The documents may come from teaching and research institutions in France or abroad, or from public or private research centers.
L'archive ouverte pluridisciplinaire HAL, est destinée au dépôt et à la diffusion de documents scientifiques de niveau recherche, publiés ou non, émanant des établissements d'enseignement et de recherche français ou étrangers, des laboratoires publics ou privés. 
See discussions, stats, and author profiles for this publication at: https://www.researchgate.net/publication/352242453

\section{Thermal transfer in amorphous superionic Li $2 \mathrm{~S}$}

Article · June 2021

DOI: 10.1103/PhysRevB.103.224204

\section{CITATION}

1

2 authors:

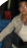

Yanguang Zhou

The Hong Kong University of Science and Technology

67 PUBLICATIONS 652 CITATIONS

SEE PROFILE

Some of the authors of this publication are also working on these related projects:

Project materials nanomechanics View project

Project Heat transfer across the interface, scattering channels. View project
READS

147

Sebastian Volz

French National Centre for Scientific Research

364 PUBLICATIONS 7,378 CITATIONS

SEE PROFILE 


\title{
Thermal transfer in amorphous superionic $\mathbf{L i}_{2} \mathrm{~S}$
}

\author{
Yanguang Zhou $\odot^{1,{ }^{*}}$ and Sebastian $\mathrm{Volz}^{2, \dagger}$ \\ ${ }^{1}$ Department of Mechanical and Aerospace Engineering, The Hong Kong University of Science and Technology, \\ Clear Water Bay, Hong Kong SAR \\ ${ }^{2}$ LIMMS/CNRS-IIS(UMI2820) Institute of Industrial Science, \\ University of Tokyo 4-6-1 Komaba, Meguro-ku Tokyo, 153-8505, Japan
}

(Received 26 May 2020; revised 22 April 2021; accepted 25 May 2021; published 8 June 2021)

\begin{abstract}
Using atomic simulations, we characterize the scattering time, the nature, and the weight of thermal excitations in the example system $\mathrm{Li}_{2} \mathrm{~S}$ from its amorphous solid state to its superionic and liquid states. For the amorphous solid state at $300 \mathrm{~K}$, the vibration scattering time was found to range from a few femtoseconds to several picoseconds. As a result, both propagons and diffusons are the main heat carriers and contribute largely to the total thermal conductivity. The enhancement of scattering among vibrations and between vibrations and the free ions' flow due to the increase of temperature leads to a large reduction of the scattering times and of the acoustic vibrational thermal conductivity, i.e., $0.8 \mathrm{~W} / \mathrm{mK}$ at $300 \mathrm{~K}$ to $0.56 \mathrm{~W} / \mathrm{mK}$ in the superionic $\mathrm{Li}_{2} \mathrm{~S}$ at $700 \mathrm{~K}$. In this latter state, the thermal conductivity contributed by convection increases to half of the total, as a result of the cross correlation between the virial heat flux and the free ions' one in liquid $\mathrm{Li}_{2} \mathrm{~S}$ at $1100 \mathrm{~K}$. The vibrational scattering time can be large $(\sim 1.5 \mathrm{ps})$ yet, and the vibrational conductivity is reduced to a still significant fraction highlighting the unexpected role of acoustic transverse and longitudinal vibrations. Our study provides a fundamental understanding of the thermal excitations at play in amorphous materials from solid to liquid, including the intermediate superionic phase.
\end{abstract}

DOI: 10.1103/PhysRevB.103.224204

\section{INTRODUCTION}

Conduction and convection heat transfer are the main heat exchange modes in solids and liquids, respectively, which is of critical importance in energy conversion [1,2], energy storage [3], and energy transport [4]. While it is well known that phonons, i.e., energy quanta of propagating lattice waves, are dominant thermal excitations in perfect crystals [5], the ones in disordered solids as well as in liquids are much more diverse and difficult to be quantitatively characterized. Thermal excitations in amorphous solids and nanostructures, e.g., amorphous Si [6-10] and polycrystalline Si nanowires [11], are classified as propagons, diffusons, and locons depending on the degree of delocalization of atomistic vibrations and on their mean free path (MFP). This description was first proposed by Allen and Feldman [6] and Cahill [12], then extended by Larkin and MacGauhey [8] and Lv and Henry [13]. The similar classification is also applied to quantify the thermal excitations in systems which are in the superionic state at high temperatures such as crystal $\mathrm{Li}_{2} \mathrm{~S}$ [14], $\mathrm{Ag}_{2} \mathrm{Te}$ [15], $\mathrm{AgCrSe}_{2}$ [16], and $\mathrm{Cu}_{3} \mathrm{SbSe}_{3}$ [17], but with an extra contribution from convection heat transfer as proved by Zhou et al. [14]. In liquids where convection heat transfer predominates, acoustic and optical phonons are experimentally observed such as in liquid Ga [18], water [19], and liquid helium [20]. Despite this progress in discovering the persistence of those modes, quantitatively characterizing the contribution

\footnotetext{
*maeygzhou@ust.hk

†volz@iis.u-tokyo.ac.jp
}

of each thermal excitation in superionic and liquid systems remains challenging and poorly documented.

In this paper, quantitative characterization of the vibrational scattering time scales and the contribution of each thermal mode from the amorphous solid to the liquid state in the example system of $\mathrm{Li}_{2} \mathrm{~S}$ was achieved by using atomistic simulations. The interval of vibrational scattering time scales is found to be large and ranging from a few femtoseconds to several picoseconds in $\mathrm{Li}_{2} \mathrm{~S}$ solids and liquids. Our analysis supports that conduction heat transfer contributed from propagons and diffusons is the dominant mode in amorphous $\mathrm{Li}_{2} \mathrm{~S}$. Those heat carriers account for one-half and one-third of the total thermal energy exchange in superionic and liquid $\mathrm{Li}_{2} \mathrm{~S}$, respectively. Convection heat transfer which results from two different mechanisms, i.e., virial atomic interactions and ions' free movement, is found to increasingly contribute to the thermal energy exchange with the fluidization of $\mathrm{Li}$ and $\mathrm{S}$ ions. Both transverse and longitudinal vibrations exist in liquid and substantially carry heat, by one-third in liquid $\mathrm{Li}_{2} \mathrm{~S}$ at $1100 \mathrm{~K}$. We would like to emphasize that we do not include the electronic thermal conductivity since the molecular dynamics simulations do not include the electronic properties of the systems.

\section{THEORETICAL MODEL}

\section{A. Thermal conductivity}

As clearly established in previous works [21], the heat current $\vec{Q}$ in a classical ionic ensemble can be written in the 
following form:

$$
\vec{Q}=\vec{Q}_{c \text {-flow }}+\vec{Q}_{\text {virial }}=\underbrace{\sum_{i}^{N} e_{i} \vec{v}_{i}}_{c \text {-flow }}+\underbrace{\frac{1}{2} \sum_{i, j ; i \neq j}^{N}\left(\vec{F}_{i j} \cdot \vec{v}_{i}\right) \vec{r}_{i j}+\sum_{i, j, k ; i \neq j \neq k}^{N}\left(\vec{F}_{i j k} \cdot \vec{v}\right)\left(\vec{r}_{i j}+\vec{r}_{i k}\right)}_{\text {virial }}
$$

in which $e_{i}$ refers to the ionic energy, $\vec{v}_{i}$ is the ionic velocity, $\vec{r}_{i j}$ is the distance between two ions $i$ and $j$, and $\vec{F}_{i j}$ and $\vec{F}_{i j k}$ represent the two-body and three-body forces, respectively. The first term in Eq. (1) results from the flow of ions and corresponds to the convection, i.e., convection heat current $\vec{Q}_{c \text {-flow. }}$. We emphasize that this heat current is resulting from the diffusion of ions in amorphous $\mathrm{Li}_{2} \mathrm{~S}$. It results from the motion of $\mathrm{Li}$ ions and from the large diffusion of other ions in $\mathrm{Li}_{2} \mathrm{~S}$. The last two terms in the right-hand side of Eq. (1) refer to and are corresponding to the interactions between two ions and are also called virial heat current $\vec{Q}_{\text {virial }}$. We have calculated the heat current components using the LAMMPS [22] with corrected heat current formula [23] updated by Boone et al. [24], and found negligible discrepancy with the results deduced from the original formula. Consequently, the thermal conductivity $\kappa$ can be divided into contributions involving ions' convection, $\kappa_{c \text {-flow }}$, interactions among ions, $\kappa_{\text {virial }}$, and a term resulting from the cross correlation between convection and conduction, $\kappa_{\text {cross }}$. Note that $\kappa_{\text {cross }}$ cannot be simply treated as the result of scattering between vibrations and liquid, but instead as the cross correlation between the convection heat current and the virial one. Physically, the virial and convection heat currents depend on interatomic forces and are derived from standard statistical mechanics. $\kappa_{\text {virial }}$ includes the contributions from propagons and diffusons, while convection is appearing in the superionic and liquid states. Both heat fluxes and their cross correlation are appearing in the Green-Kubo formula [25]:

$\kappa=\kappa_{\text {virial }}+\kappa_{c \text {-flow }}+\kappa_{\text {cross }}=\frac{1}{3 k_{b} V T^{2}} \int_{0}^{\infty}[\underbrace{\left\langle\vec{Q}_{\text {virial }}(t) \cdot \vec{Q}_{\text {virial }}(0)\right\rangle}_{\text {virial }}+\underbrace{\left\langle\vec{Q}_{c \text {-flow }}(t) \cdot \vec{Q}_{c \text {-flow }}(0)\right\rangle}_{c \text {-flow }}+\underbrace{2\left\langle\vec{Q}_{\text {virial }}(t) \cdot \vec{Q}_{c \text {-flow }}(0)\right\rangle}_{\text {cross }}] d t$,

where $k_{b}$ is the Boltzmann constant, $V$ is the system volume, $T$ represents the system temperature, and $t$ denotes the autocorrelation time. The angular bracket indicates ensemble averaging.

\section{B. Simulations}

A $\mathrm{Li}_{2} \mathrm{~S}$ crystal including 6144 atoms with interactions depicted by the reactive force-field (ReaxFF) [26] was first equilibrated at a high-temperature liquid state, i.e., $3000 \mathrm{~K}$, for $62.5 \mathrm{ps}$ and then cooled down to $300 \mathrm{~K}$ in the $N-P-T$ (constant particle number, pressure, and temperature) ensemble with a cooling rate of $10 \mathrm{~K} / \mathrm{ps}$. The structure is finally equilibrated at $300 \mathrm{~K}$ for another $62.5 \mathrm{ps}$ in the $N-V-T$ (constant particle number, volume, and temperature) ensemble to reduce the metastabilities. Next, the generated amorphous $\mathrm{Li}_{2} \mathrm{~S}$ was annealed from $300 \mathrm{~K}$ to $1500 \mathrm{~K}$ for $37.5 \mathrm{ps}$ in the $N-V-T$ ensemble to simulate the amorphous, superionic, and liquid $\mathrm{Li}_{2} \mathrm{~S}$. The heat current which is used as an input in thermal conductivity calculations based on Green-Kubo theory [25] was computed for $12.5 \mathrm{ps}$ in the $N-V-E$ (constant particle number, volume, and energy) ensemble. The time step in all the simulations is $0.25 \mathrm{fs}$. Periodic boundary conditions were applied in all three directions. Amorphous $\mathrm{Li}_{2} \mathrm{~S}$ at $300 \mathrm{~K}$ with $4 \times 4 \times 4,6 \times 6 \times 6$, and $8 \times 8 \times 8$ unit cells which correspond to 768,2592 , and 6144 ions in the systems are used as examples to calibrate the size effect on Green-Kubo thermal conductivity (GKTC). From Fig. 1(a), we can find that the size effect can be ignored when the number of the ions in the system reaches 2592 . The thermal conductivity reported in the following corresponds to the system with 6144 ions. Figures 1(b)-1(d) show the Green-Kubo thermal conductivity of amorphous $\mathrm{Li}_{2} \mathrm{~S}$ at various temperatures as a function of the autocorrelation time taken as an upper limit for the time integration in the Green-Kubo formula. Figures 1(b)-1(d) reveal that an autocorrelation time of $10 \mathrm{ps}$ is yielding a reasonably well converged thermal conductivity. It should be noted that the thermal conductivity is a collective property of atomistic long range trajectories taking the form of a convective flow and atomic interactions. As suggested by Ladd et al. [27], Volz et al. [28,29], and McGaughey and Kaviany [30], the relaxation time $\tau$ of heat carriers is related to the heat current $\vec{Q}(t)$ autocorrelation function (HCACF)

$$
H=\langle\vec{Q}(t) \cdot \vec{Q}(0)\rangle .
$$

The HCACF is then normalized by its zero-time value to allow for comparisons between the results at different temperatures. Unlike the HCACF of crystals, which follows a single stage exponential decay [30], our results show that the HCACF in our three typical amorphous superionic systems drops below zero and then oscillates between positive and negative values as it converges to zero when the correlation time is equal to about 0.5 ps [Figs. 1(b)-1(d)]. This behavior has been widely observed in other amorphous materials [30-32] because each atom has a distinct local environment as suggested by McGaughey and Kaviany [30]. The short time, i.e., around $0.5 \mathrm{ps}$, required by the HCACF to converge to zero eventually means that the scattering process among all the heat carriers will bring an equilibrium state during this period. Consequently, the resulting thermal conductivity for 

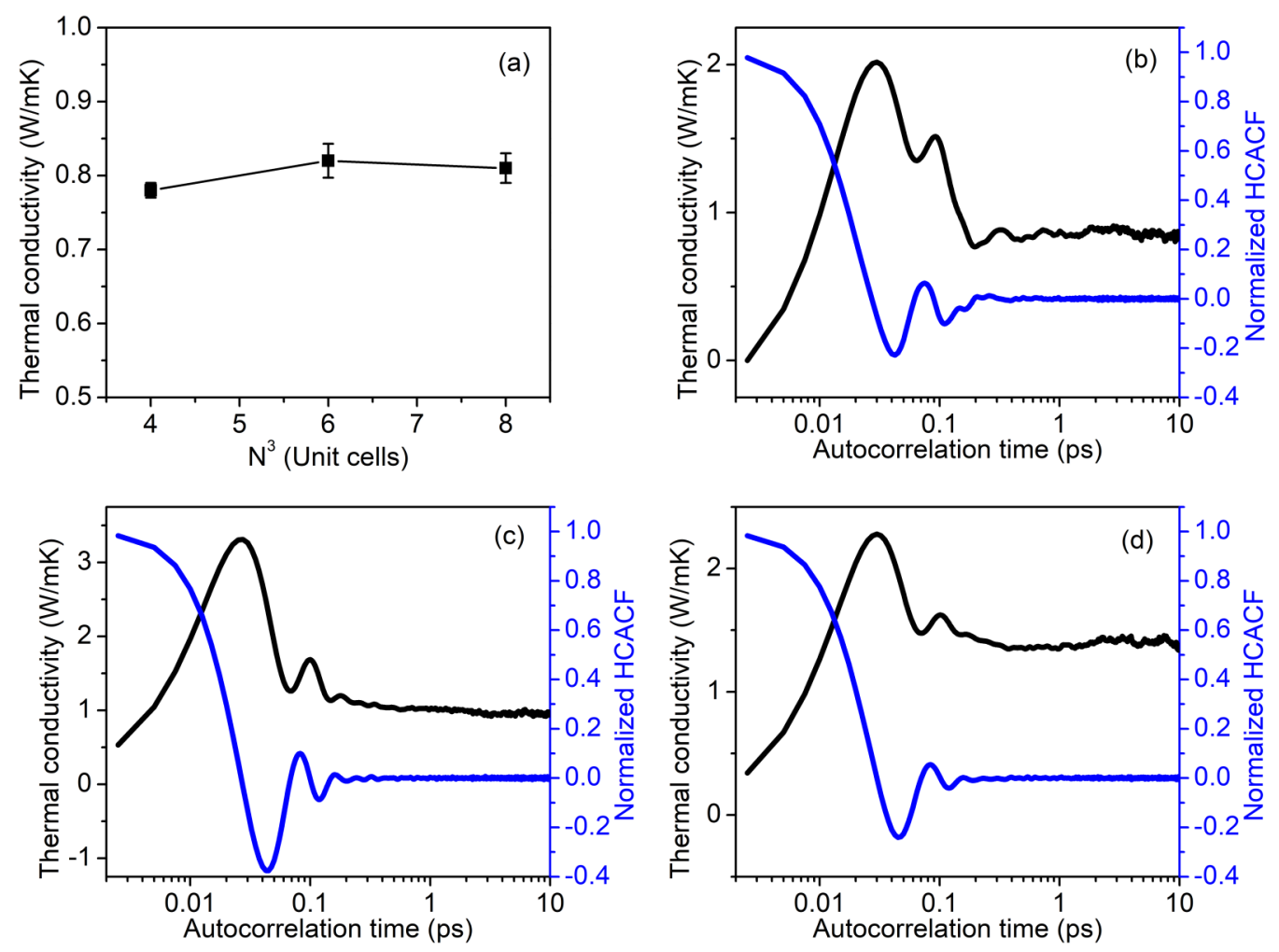

FIG. 1. (a) Size effect on thermal conductivity of amorphous $\mathrm{Li}_{2} \mathrm{~S}$ at $300 \mathrm{~K}$; $\mathrm{N}$ is the number of unit cells along one direction. The normalized autocorrelation function (blue line) and the corresponding thermal conductivity (black line) for amorphous superionic $\mathrm{Li}_{2} \mathrm{~S}$ at (b) $300 \mathrm{~K}$, (c) $700 \mathrm{~K}$, and (d) $1100 \mathrm{~K}$.

the amorphous superionic $\mathrm{Li}_{2} \mathrm{~S}$ which is an integration of the HCACF converges to a stable value when the autocorrelation time reaches around $0.5 \mathrm{ps}$. For comparison, the average vibrational relaxation times are $0.31 \mathrm{ps}, 0.27 \mathrm{ps}, 0.21 \mathrm{ps}$ at $300 \mathrm{~K}, 700 \mathrm{~K}$, and $1100 \mathrm{~K}$, respectively, as shown in the below analysis. Since the vibrations contribute to a large fraction of the total thermal conductivity $(92 \%$ at $300 \mathrm{~K}, 61 \%$ at $700 \mathrm{~K}$, and $37 \%$ at $1100 \mathrm{~K}$ ), we may also infer that the scattering process among the heat carriers reaches the equilibrium state in a very short time smaller than $1 \mathrm{ps}$.

We also calculate the thermal conductivity using the Einstein formula [33]

$$
\kappa=\frac{1}{3 k_{b} V T^{2}} \lim _{t \rightarrow \infty} \frac{1}{2 t}\left\langle[\vec{R}(t)-\vec{R}(0)]^{2}\right\rangle,
$$

in which $\vec{R}(t)$ is the integrated heat current and can be calculated using

$$
\vec{R}(t)=\int_{0}^{t} \vec{Q}(t) d t
$$

Our results calculated using this expression are reported in Fig. 2 and clearly show that the autocorrelations in the three example systems follow linear time dependences when the autocorrelation time is larger than $0.5 \mathrm{ps}$, and the corresponding thermal conductivities are $0.84 \mathrm{~W} / \mathrm{mK}$ at $300 \mathrm{~K}, 0.94 \mathrm{~W} / \mathrm{mK}$ at $700 \mathrm{~K}$, and $1.42 \mathrm{~W} / \mathrm{mK}$ at $1100 \mathrm{~K}$, which satisfactorily agrees with the computed Green-Kubo formula.

\section{Dynamical structure factor and pseudodispersion relations}

To gain more insight into the contribution to heat exchange from each thermal excitation, we calculate the vibrational dispersions of $\mathrm{Li}_{2} \mathrm{~S}$ using the dynamical structure factor $S$, given by [34]

$$
\begin{aligned}
S_{T \text { or } L}(|\vec{q}|, \omega)= & \int_{0}^{\infty}\left\langle\vec{E}_{T \text { or } L}(|\vec{q}|, t) \cdot \vec{E}_{T \text { or } L}(-|\vec{q}|, 0)\right\rangle \\
& \times \exp (-i \omega t) d t,
\end{aligned}
$$

where $\omega$ refers to the angular frequency of the lattice vibrations. $\vec{q}$ corresponds to the wave vector and is computed

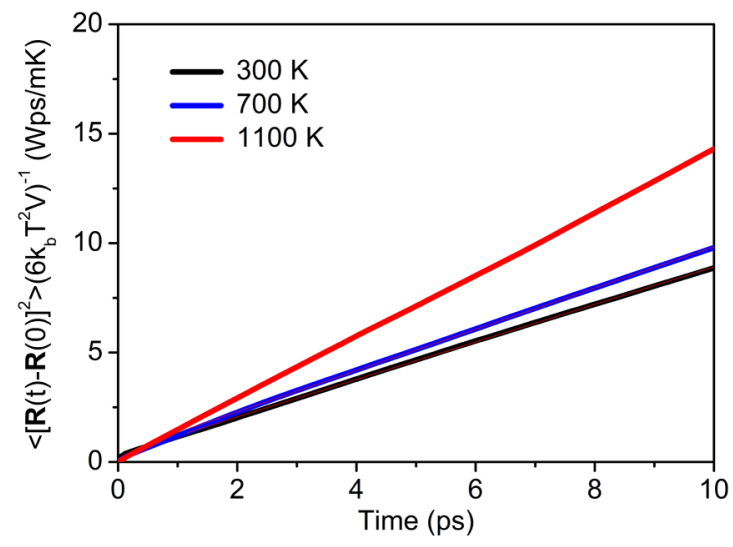

FIG. 2. Thermal conductivity multiplied by time according to Einstein relation for amorphous $\mathrm{Li}_{2} \mathrm{~S}$ at $300 \mathrm{~K}$ (black line), $700 \mathrm{~K}$ (blue line), and $1100 \mathrm{~K}$ (red line). 

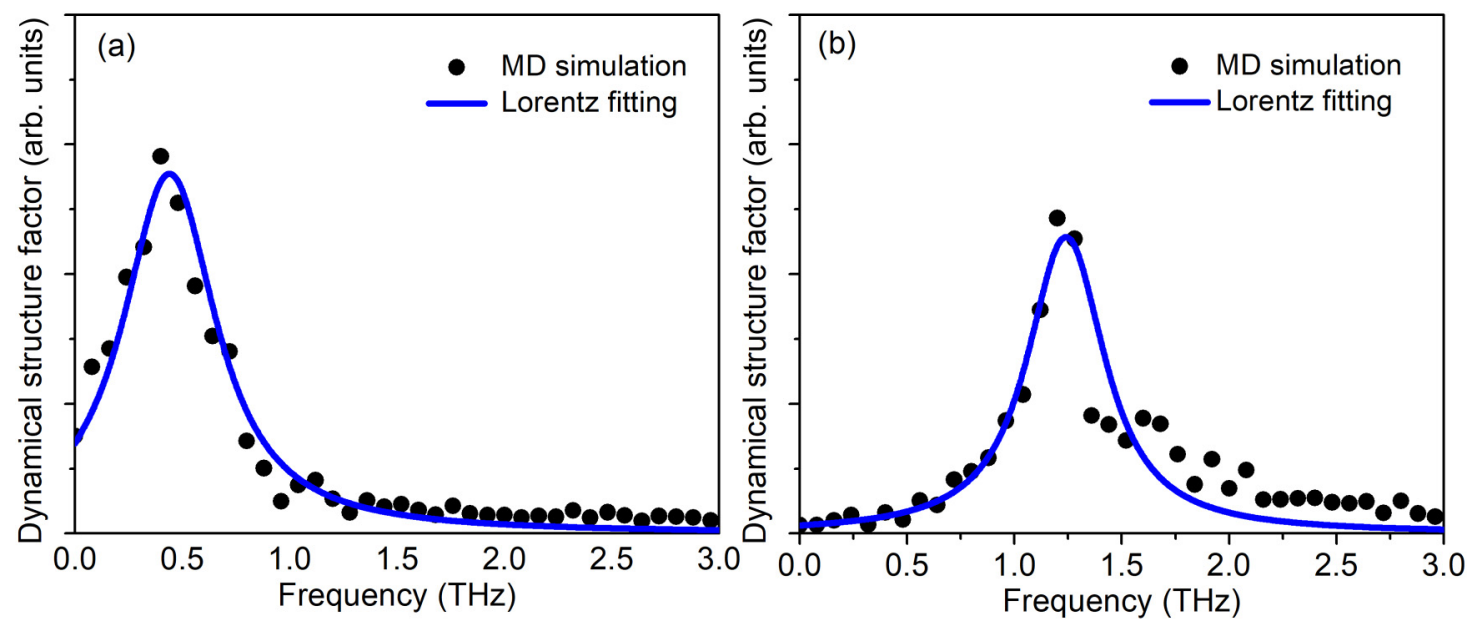

FIG. 3. Dynamical structure factor for the transverse vibrational mode (a) and longitudinal vibrational mode (b) for the mode with wavevector $(2 \pi / L x, 0,0)$ at $1100 \mathrm{~K}$.

using

$$
\vec{q}=\frac{2 \pi n_{x}}{L x} \vec{i}+\frac{2 \pi n_{y}}{L y} \vec{j}+\frac{2 \pi n_{z}}{L z} \vec{k},
$$

in which $n_{x}$ or $y$ or $z$ is an integer and should be smaller than $L_{x}$ or $y$ or $z / a_{\min }$, where $L_{x}$ or $y$ or $z$ and $a_{\min }$ are system length along $x$ or $y$ or $z$ directions and the minimal interatomic space, respectively. $\vec{E}_{T}$ and $\vec{E}_{L}$ stand for the mode velocities related to transverse and longitudinal polarizations, respectively,

$$
\begin{gathered}
\vec{E}_{T}(|\vec{q}|, t)=\sum\left\{\vec{v}_{i}(t)-\left[\vec{v}_{i}(t) \cdot \hat{\vec{q}}\right] \hat{\vec{q}}\right\} \exp \left[i \vec{q} \cdot \vec{r}_{i}(t)\right], \\
\vec{E}_{L}(|\vec{q}|, t)=\sum\left[\vec{v}_{i}(t) \cdot \hat{\vec{q}}\right] \hat{\vec{q}} \exp \left[i \vec{q} \cdot \vec{r}_{i}(t)\right],
\end{gathered}
$$

where $\hat{\vec{q}}=\vec{q} /|\vec{q}|$, and $\vec{r}_{i}$ refers to the position of the ion with index $i$. The structure factor has been successfully used to depict the dispersion relations in disordered solids and liquids $[7,18,34]$. For its calculations, all the simulations start with 62.5 ps $N-P-T$ relaxation to allow the systems to reach the equilibrium state, and then the atomistic velocity and the position, which are the inputs for Eqs. (6), (8), and (9), are dumped every $0.0125 \mathrm{ps}$ in the next $31.25 \mathrm{ps} N-V-E$ simulation. The time step is $0.25 \mathrm{fs}$ and the periodic boundary conditions are implemented in all three directions. Following the discussion above, we calculate the dynamical structure factors of the three typical systems, i.e., amorphous solid $\mathrm{Li}_{2} \mathrm{~S}$ at $300 \mathrm{~K}$, superionic $\mathrm{Li}_{2} \mathrm{~S}$ at $700 \mathrm{~K}$, and liquid $\mathrm{Li}_{2} \mathrm{~S}$ at $1100 \mathrm{~K}$. A typical dynamical structure factor for the wave vector $(2 \pi / L x, 0,0)$ at $1100 \mathrm{~K}$ is shown in Fig. 3. A Gaussian window function of $0.25 \mathrm{THz}$ is applied in the Lorentz function to fit the simulation data.

We further calculate the mode relaxation time $\tau$ of vibrations by fitting the dynamical structure factor with the Lorentz function

$$
S=\frac{I_{p}}{\left[\left(\omega-\omega_{p}\right) / \Delta\right]^{2}+1},
$$

where $I_{p}$ and $\omega_{p}$ are the magnitude and the frequency at the peak center, respectively. The linewidth $\Delta$ is equal to half of the scattering rate $\Gamma$ and the relaxation time can then be calculated as $\tau=1 / 2 \Delta$.
We emphasize that the effective wave vector as defined in Eq. (7) is an equivalent wave vector of an imaginary crystalline structure with a lattice constant $a_{\text {min }}$. The dispersion relation shown in Fig. 4 then is the vibrational relation between the effective wave vector and the vibrational frequency. This dispersion is equivalent to the phonon dispersion in the crystals, which are fitted using the data below $8 \mathrm{THz}$ via the exponential functions. These fitting functions are given in Table I.

\section{Heat carriers landscape}

Atomic vibrations related to thermal conductivity can be calculated using

$$
\kappa_{\text {vibration }}=\sum_{|\vec{q}|} c_{V}(|\vec{q}|) v_{g}(|\vec{q}|)^{2} \tau(|\vec{q}|),
$$

where $c_{V}(|\vec{q}|)$ refers to the volumetric heat capacity which is equal to $k_{b} / V, v_{g}(|\vec{q}|)$ to the group velocity, and $\tau(|\vec{q}|)$ refers to the relaxation time of the atomic vibrations. We can then calculate the effective mean free path $\Lambda_{\text {eff }}(|\vec{q}|)$ using $\Lambda_{\text {eff }}(|\vec{q}|)=v_{g} \tau(|\vec{q}|)$.

To determine the minimal interatomic distance, we calculate the radial distribution function $g(r)$ of amorphous $\mathrm{Li}_{2} \mathrm{~S}$ [Fig. 5(a)]. Although bonds with lengths of 0.22 to $0.24 \mathrm{~nm}$ exist in amorphous $\mathrm{Li}_{2} \mathrm{~S}$, we select the bond corresponding to the peak at $0.24 \mathrm{~nm}$ as the minimal interatomic distance since it is predominant over the $0.22 \mathrm{~nm}$ one [Fig. 5(b)]. These vibrations with effective mean free paths smaller than the minimal interatomic space $0.24 \mathrm{~nm}$ are regarded as diffusons and the others as propagons. The corresponding thermal conductivities shall hence be written in the following forms:

$$
\begin{aligned}
\kappa_{\text {diffuson }} & =\sum_{\Lambda_{\text {eff }}(|\vec{q}|)<0.24 \mathrm{~nm}} c_{V}(|\vec{q}|) v_{g}(|\vec{q}|) \Lambda_{\mathrm{eff}}(|\vec{q}|), \\
\kappa_{\text {propagon }} & =\sum_{\Lambda_{\mathrm{eff}}(|\vec{q}|)>0.24 \mathrm{~nm}} c_{V}(|\vec{q}|) v_{g}(|\vec{q}|) \Lambda_{\mathrm{eff}}(|\vec{q}|) .
\end{aligned}
$$

Note that using $0.22 \mathrm{~nm}$ as the minimal interatomic distance would also decrease (increase) the thermal conductivity contributed from diffusons (propagons) by only $0.01 \mathrm{~W} / \mathrm{mK}$ 

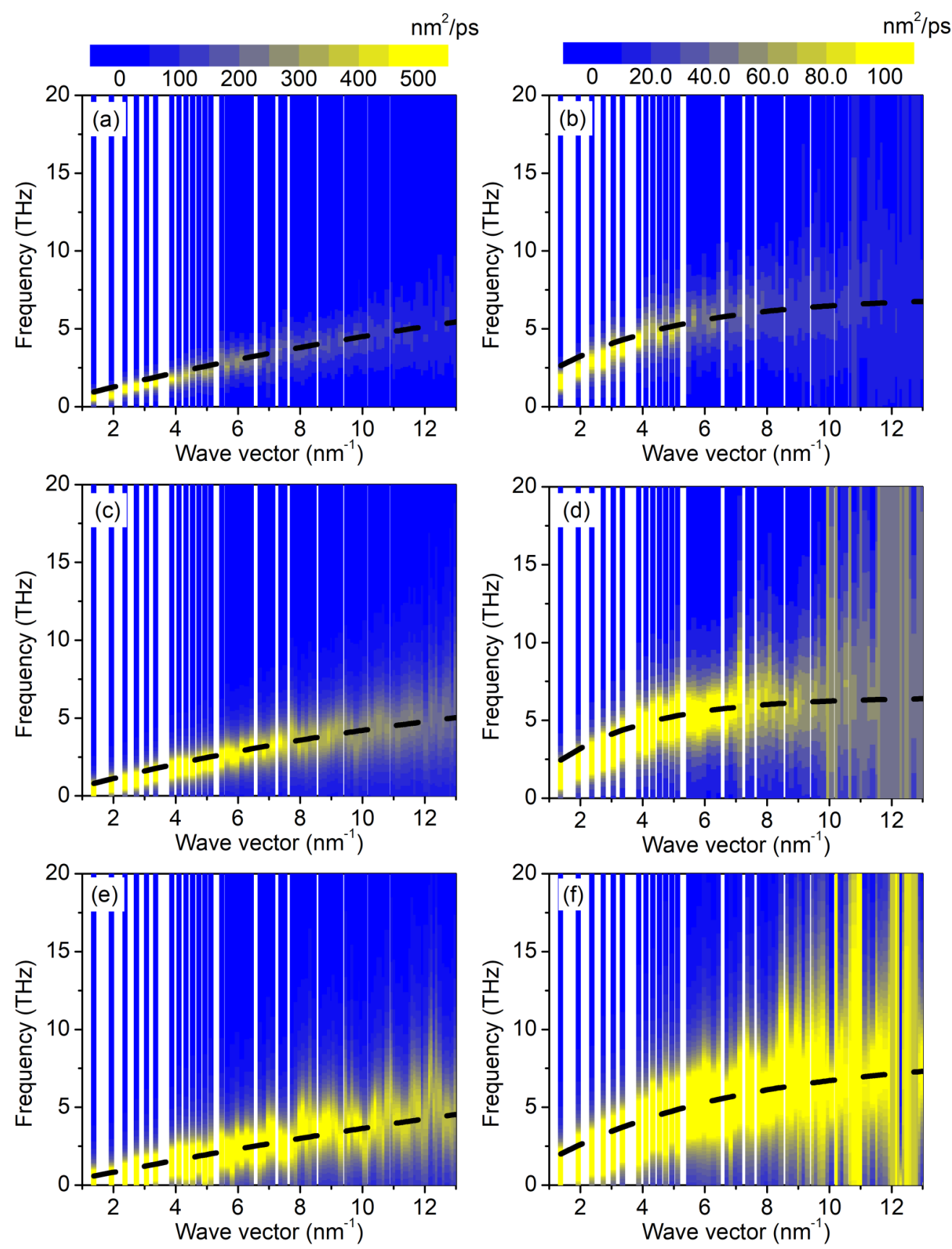

FIG. 4. Structure factors for the transverse branch at (a) $300 \mathrm{~K}$, (c) $700 \mathrm{~K}$, and (e) $1100 \mathrm{~K}$ and the longitudinal branch at (b) $300 \mathrm{~K}$, (d) $700 \mathrm{~K}$, and (f) $1100 \mathrm{~K}$. The black dashed lines are fitted dispersions.

in the three example systems. The atomic vibrations are resulting from the interactions between two ions, and therefore $\kappa_{\text {vibration }}$ is equal to $\kappa_{\text {virial }}$ in solid amorphous $\mathrm{Li}_{2} \mathrm{~S}$. However, the interactions between two ions in the superionic and liquid $\mathrm{Li}_{2} \mathrm{~S}$ systems not only contribute to conduction heat transfer but also to convection heat transfer. This latter can be by

TABLE I. Fitting functions used to fit the vibrational dispersions as shown in Fig. 4.

\begin{tabular}{lll}
\hline \hline Temperature $(\mathrm{K})$ & Fitting function for transverse branch & Fitting function for longitudinal branch \\
\hline 300 & $\omega(|\vec{q}|)=-11.52 \exp \left(-\frac{|\vec{q}|}{21.7}\right)+11.77$ & $\omega(|\vec{q}|)=-6.1 \exp \left(-\frac{|\vec{q}|}{4.19}\right)+6.33$ \\
700 & $\omega(|\vec{q}|)=-8.84 \exp \left(-\frac{|\vec{q}|}{15.85}\right)+8.92$ & $\omega(|\vec{q}|)=-6.32 \exp \left(-\frac{|\vec{q}|}{3.04}\right)+6.47$ \\
1100 & $\omega(|\vec{q}|)=-14.32 \exp \left(-\frac{|\vec{q}|}{34.24}\right)+14.35$ & $\omega(|\vec{q}|)=-7.8 \exp \left(-\frac{|\vec{q}|}{6.16}\right)+8.25$ \\
\hline \hline
\end{tabular}



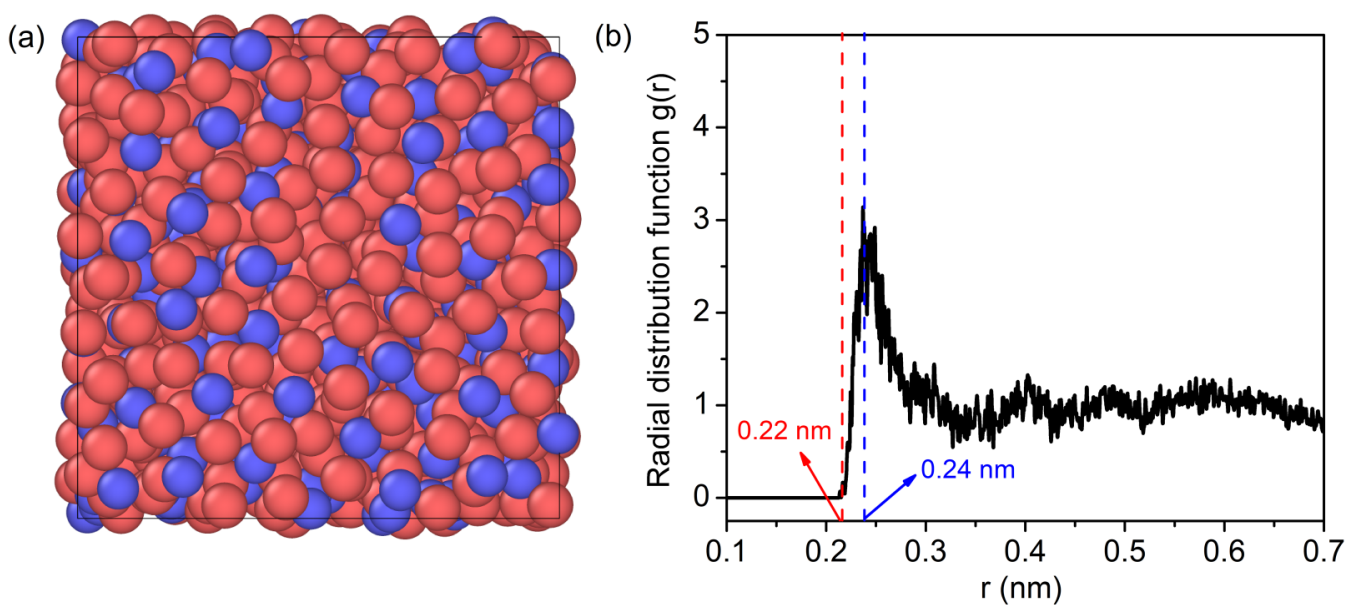

FIG. 5. (a) Structure of amorphous $\mathrm{Li}_{2} \mathrm{~S}$; red spheres are $\mathrm{Li}$ ions and blue spheres are the $\mathrm{S}$ ions. (b) The radial distribution function $g(r)$ of amorphous $\mathrm{Li}_{2} \mathrm{~S}$.

subtraction via

$$
\kappa_{c \text {-virial }}=\kappa_{\text {virial }}-\kappa_{\text {vibration }}=\kappa_{\text {virial }}-\kappa_{\text {diffuson }}-\kappa_{\text {propagon }} .
$$

\section{RESULTS AND DISCUSSIONS}

\section{A. Green-Kubo thermal conductivity}

To analyze the thermal transport behavior in $\mathrm{Li}_{2} \mathrm{~S}$, we first calculate the atomic trajectories and the diffusion coefficient. The time trajectories of $\mathrm{Li}$ and $\mathrm{S}$ ions at three representative temperatures, i.e., $300 \mathrm{~K}, 700 \mathrm{~K}$, and $1100 \mathrm{~K}$, are projected onto the [100] lattice plane (Fig. 6) as the superposition of many frames at different times uncovering the range in which ions can travel in real space. At low temperatures, i.e., $300 \mathrm{~K}$, both $\mathrm{Li}$ and $\mathrm{S}$ ions vibrate around their equilibrium positions [Figs. 6(a) and 6(b)]. At a medium temperature of $700 \mathrm{~K}$, although $\mathrm{S}$ ions still vibrate around their original sites, $\mathrm{Li}$ ions can travel far away from their equilibrium positions and flow across the system [Figs. 6(c) and 6(d)], indicating that they can be treated as in a liquid phase. At the high temperature of $1100 \mathrm{~K}$, both Li and S ions can flow freely [Figs. 6(e) and 6(f)].

The diffusion coefficient is evaluated via fitting the mean square displacement (MSD) of Li and S ions' trajectories in reaxFF equilibrium molecular dynamics (EMD) simulations [Figs. 7(a) and 7(b)] as [33]

$$
M(t)=\frac{1}{6 N_{a}}\left\langle\sum_{i=1}^{N_{a}}\left[\vec{r}_{i}(t)-\vec{r}_{i}(0)\right]^{2}\right\rangle,
$$
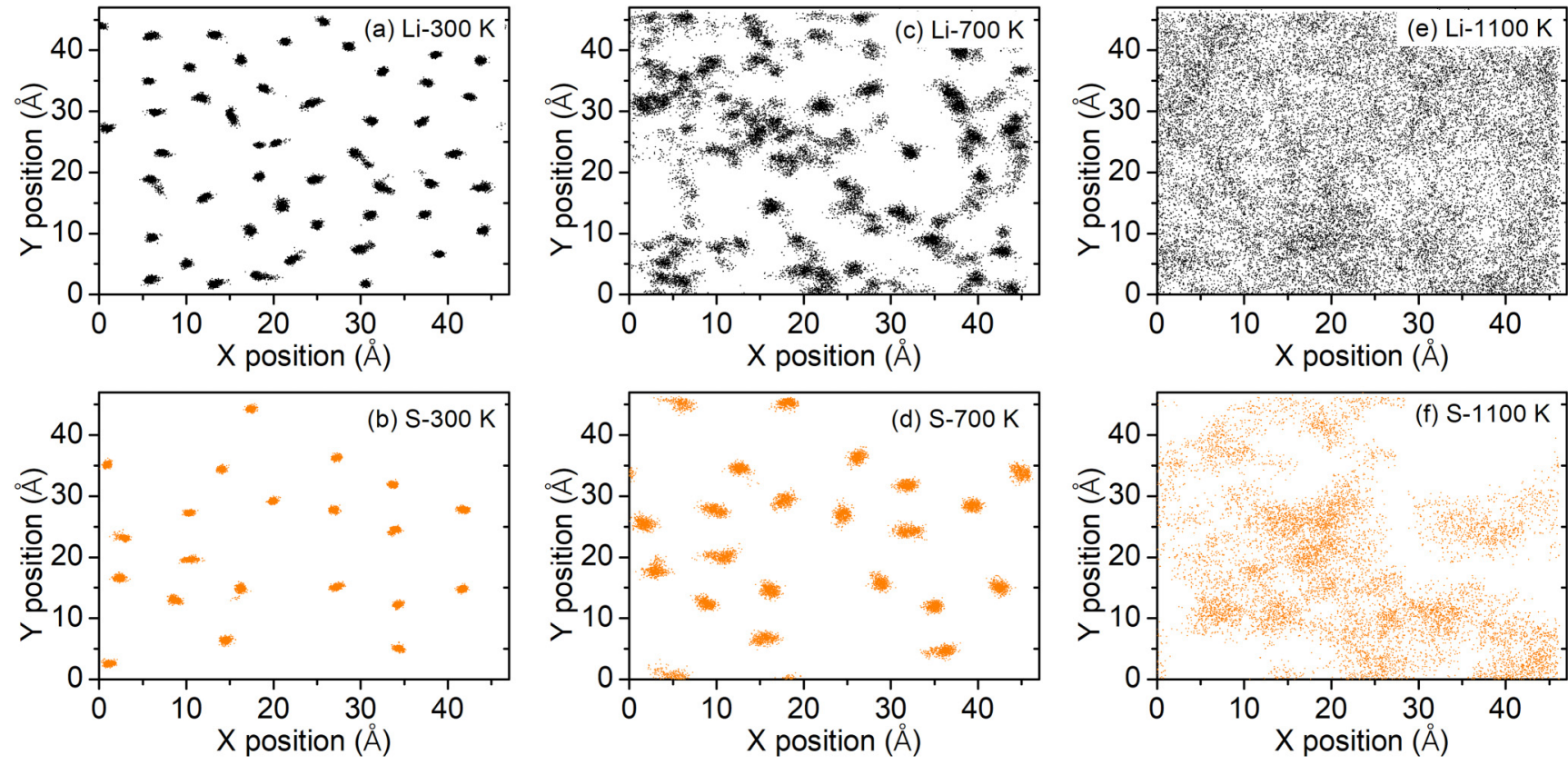

FIG. 6. Trajectories of Li ions (gray) and S ions (orange) at (a),(b) $300 \mathrm{~K}$, (c),(d) $700 \mathrm{~K}$, and (e),(f) $1100 \mathrm{~K}$ from the reactive force-field molecular dynamic simulations. 

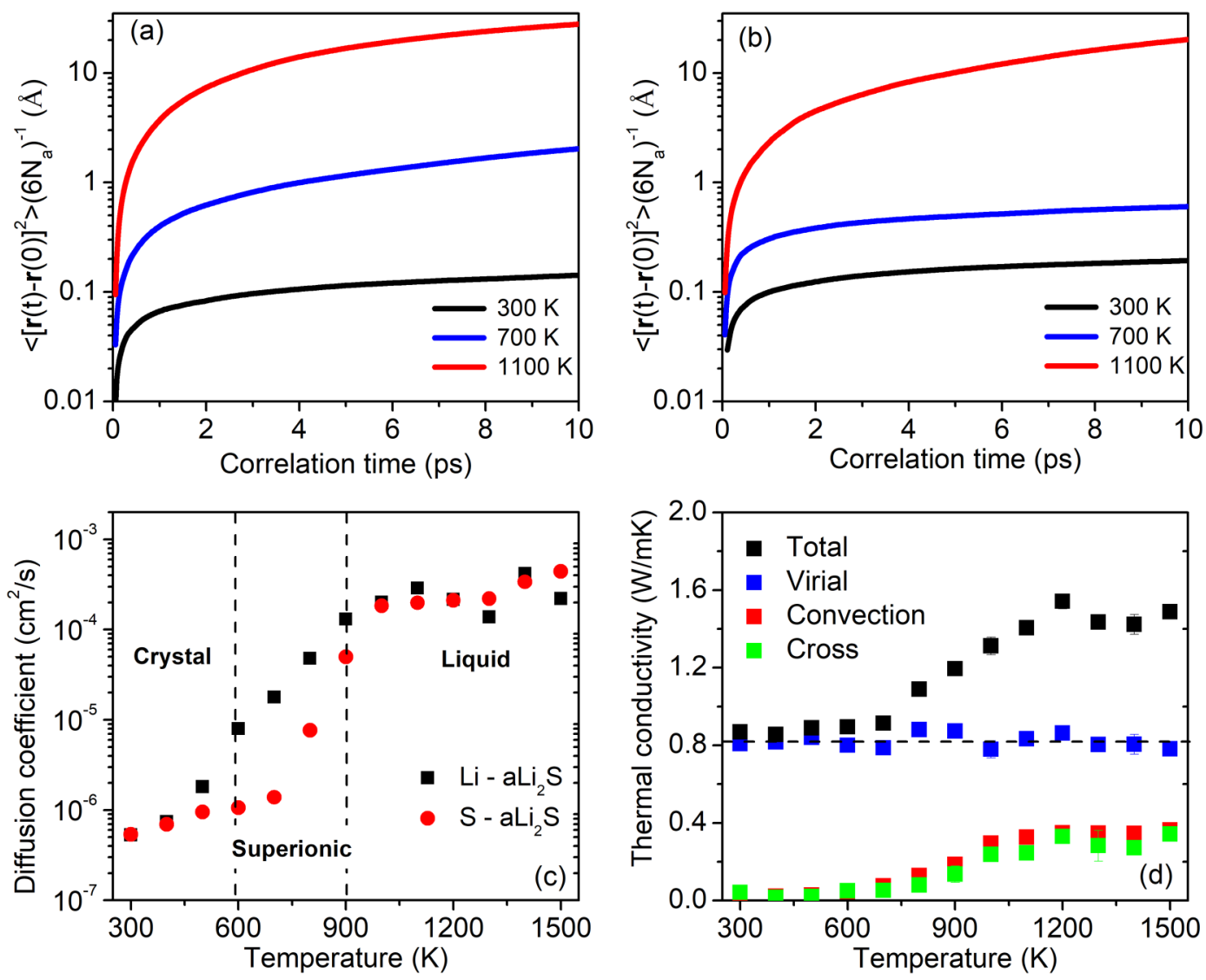

FIG. 7. Einstein relation based diffusion coefficient for Li (a) and S (b) at $300 \mathrm{~K}$ (black line), $700 \mathrm{~K}$ (blue line), and $1100 \mathrm{~K}$ (red line). (c) Diffusion coefficient of Li (black dots) and S (red dots) ions at different temperatures. (d) Thermal conductivity contributed from the virial term (blue dots), the convection term (red dots), and the cross term (green dots) computed using Eq. (2).

where $t$ is the lag time, $\vec{r}_{i}$ is the position of atom $i$, and $N_{a}$ is the number of $\mathrm{Li}$ or $\mathrm{S}$ ions. The diffusion coefficient can then be calculated using the Einstein relation

$$
D=\lim _{t \rightarrow \infty} \frac{M(t)}{t}
$$

According to the diffusion coefficients of $\mathrm{Li}$ and $\mathrm{S}$ ions reported in Fig. 7(c) and to the atomic trajectories shown in Fig. 6, amorphous $\mathrm{Li}_{2} \mathrm{~S}$ is found to change from the solid state to the superionic state at the transition temperature of $600 \mathrm{~K}$ and further to the liquid state at the critical temperature of $800 \mathrm{~K}$ [Fig. 7(c) and Fig. 6]. Below $600 \mathrm{~K}$, both $\mathrm{Li}$ and $\mathrm{S}$ ions manifest solidlike mobilities [35]. When the temperature of the system is in the range of $600-800 \mathrm{~K}$, the diffusion coefficient of $\mathrm{S}$ deviates significantly from that of $\mathrm{Li}$, and the $\mathrm{Li}$ ions vibrate far away from their equilibrium positions [Fig. 7(c) and Fig. 6]. Above $800 \mathrm{~K}$, both $\mathrm{Li}$ and $\mathrm{S}$ ions manifest liquidlike mobilities with high diffusion
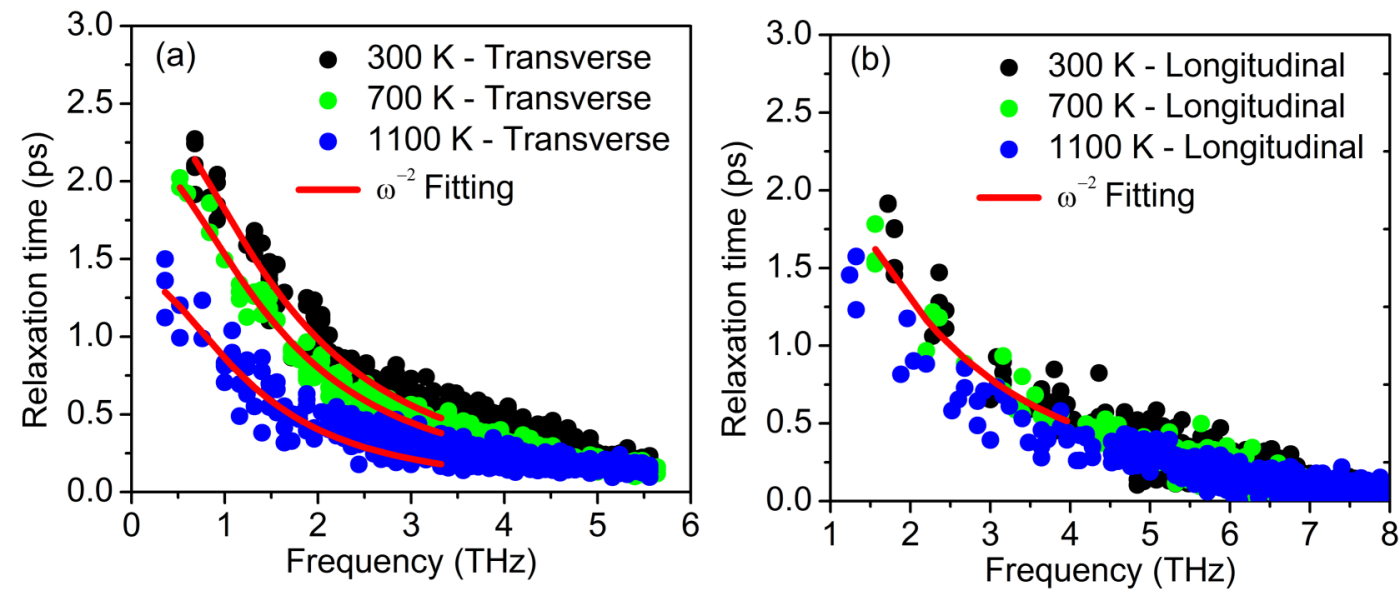

FIG. 8. Relaxation times of (a) the transverse branch and (b) the longitudinal branch for $\mathrm{Li}_{2} \mathrm{~S}$ at $300 \mathrm{~K}, 700 \mathrm{~K}$, and $1100 \mathrm{~K}$. 

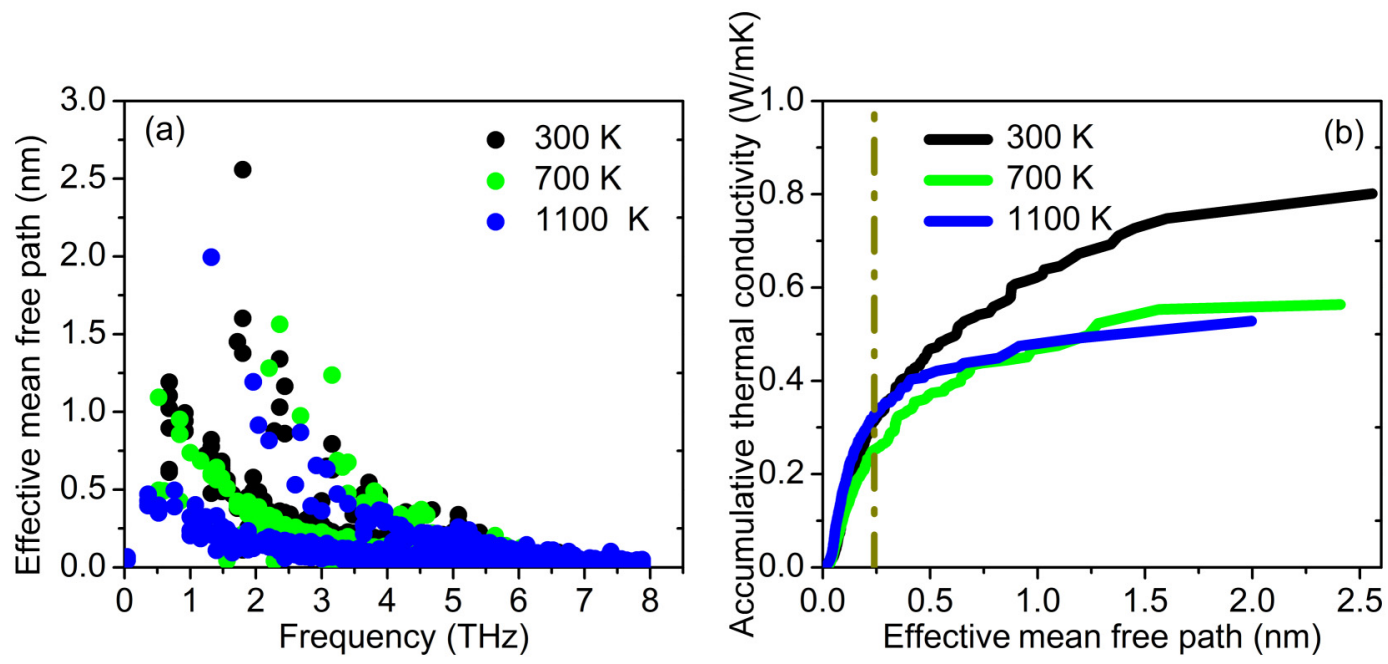

FIG. 9. (a) Effective MFP and (b) accumulative thermal conductivity with respect to the effective $\mathrm{MFP}$ for $\mathrm{Li}_{2} \mathrm{~S}$ at $300 \mathrm{~K}, 700 \mathrm{~K}$, and $1100 \mathrm{~K}$.

coefficients [35] [Fig. 7(c) and Fig. 6]. Figure 7(d) shows the temperature-dependent thermal conductivity of the systems and the corresponding contributions from the virial atomic interactions, convection, and cross terms. The thermal conductivity contributed by convection and cross terms is found to be negligible when amorphous $\mathrm{Li}_{2} \mathrm{~S}$ is in solid state at temperatures below $600 \mathrm{~K}$, but to increase gradually in superionic and liquid phases when the temperature is ranging from $600 \mathrm{~K}$ to $1100 \mathrm{~K}$. Thermal conductivity then further reaches a plateau due to the saturation of the diffusion coefficient [Fig. 7(c)] when the system temperature exceeds $1100 \mathrm{~K}$.

\section{B. Vibrational scattering processes}

To gain more insight into the contribution to heat exchange from each thermal excitation, we calculate the vibrational dispersions of the three typical systems, i.e., amorphous solid $\mathrm{Li}_{2} \mathrm{~S}$ at $300 \mathrm{~K}$, superionic $\mathrm{Li}_{2} \mathrm{~S}$ at $700 \mathrm{~K}$, and liquid $\mathrm{Li}_{2} \mathrm{~S}$ at $1100 \mathrm{~K}$ using the dynamical structure factor $S$. As revealed by Fig. 4, clearly defined dispersions exist up to $5 \mathrm{THz}$ for the transverse branch and to $7.5 \mathrm{THz}$ for the longitudinal one. At $300 \mathrm{~K}$, since both $\mathrm{Li}$ and $\mathrm{S}$ ions have solidlike mobilities, transverse [Fig. 4(a)] and longitudinal [Fig. 4(b)] acoustic dispersions are observed in the systems with small frequency broadenings. By increasing the system temperature to $700 \mathrm{~K}$ [Figs. 4(c) and 4(d)], Li ions flow in the system and atomic vibrations still carry a substantial amount of heat (see analysis below). More interestingly, the scattering between the flowing $\mathrm{Li}$ ions and the longitudinal acoustic modes is found to be much stronger than that occurring with the transverse acoustic modes. Transverse acoustic modes are related to the shear stress among ions and the longitudinal modes are the consequence of normal stress between two ions. When $\mathrm{Li}$ ions flow, the shear stress between a specific $\mathrm{Li}$ ion and other ions becomes weaker and thus the scattering of $\mathrm{Li}$ ions with transverse modes is weak compared to that on the longitudinal modes. By further increasing the system temperature to $1100 \mathrm{~K}$, both $\mathrm{Li}$ and $\mathrm{S}$ ions enter the liquid state [Fig. 7(c)]. Although the scattering among different heat carriers, which are vibrations and ions' flow, is quite strong, there are still propagating modes existing in liquid $\mathrm{Li}_{2} \mathrm{~S}$ [Figs. 4(e) and 4(f)] and heat conduction largely contributes to the total thermal energy exchange.

The clear $\omega^{-2}$ scaling below $2.5 \mathrm{THz}$ for the transverse branch [red line in Fig. 8(a)] and below $4 \mathrm{THz}$ for the longitudinal branch [red line in Fig. 8(b)] indicates that propagating modes exist in the systems [8] and that their contributions to thermal conductivity are substantial. As discussed above, longitudinal vibrations undergo a larger number of scattering events and therefore the relaxation times of the transverse modes are generally larger than the ones of the longitudinal modes in the low-frequency region. With the increase of the system temperature, from $300 \mathrm{~K}$ to $700 \mathrm{~K}(1100 \mathrm{~K})$, the relaxation time becomes smaller due to the increase of Umklapp scattering and to the interaction between vibrations and the Li-ions flow [14], since both $\mathrm{Li}$ and $\mathrm{S}$ ions flow at $1100 \mathrm{~K}$ [Fig. 7(c)].

\section{Effective mean free path}

We then move to the estimation of the effective MFP $\Lambda_{\text {eff }}$ of the vibrational modes [Fig. 9(a)]. To calculate this quantity, we first estimate the group velocity $v_{g}=d \omega / d k$ using the fitted dispersions (black dashed lines in Fig. 4) and write $\Lambda_{\text {eff }}=v_{g} \tau$. As shown in Fig. 9(a), the effective MFPs of a large amount of vibrations in the low-frequency region are larger than $0.24 \mathrm{~nm}$ corresponding to the distance between nearest neighboring ions and the effective MFP of a fraction of the low-frequency vibrations can be as large as $2.5 \mathrm{~nm}$ at $300 \mathrm{~K}(2.2 \mathrm{~nm}$ at $700 \mathrm{~K}$ and $2.0 \mathrm{~nm}$ at $1100 \mathrm{~K})$. As suggested by Figs. 8(a) and 8(b), those latter MFPs confirm that a large population of the low-frequency vibrations consists of propagating modes that can be assimilated to propagons. The vibrations with effective MFPs smaller than $0.24 \mathrm{~nm}$ cannot be treated as plane waves anymore since their wavelength cannot be defined on the atomic lattice. They are consequently categorized as diffusons in agreement with the theory of Allen and Feldman [6]. Diffusons carry energy through harmonic 


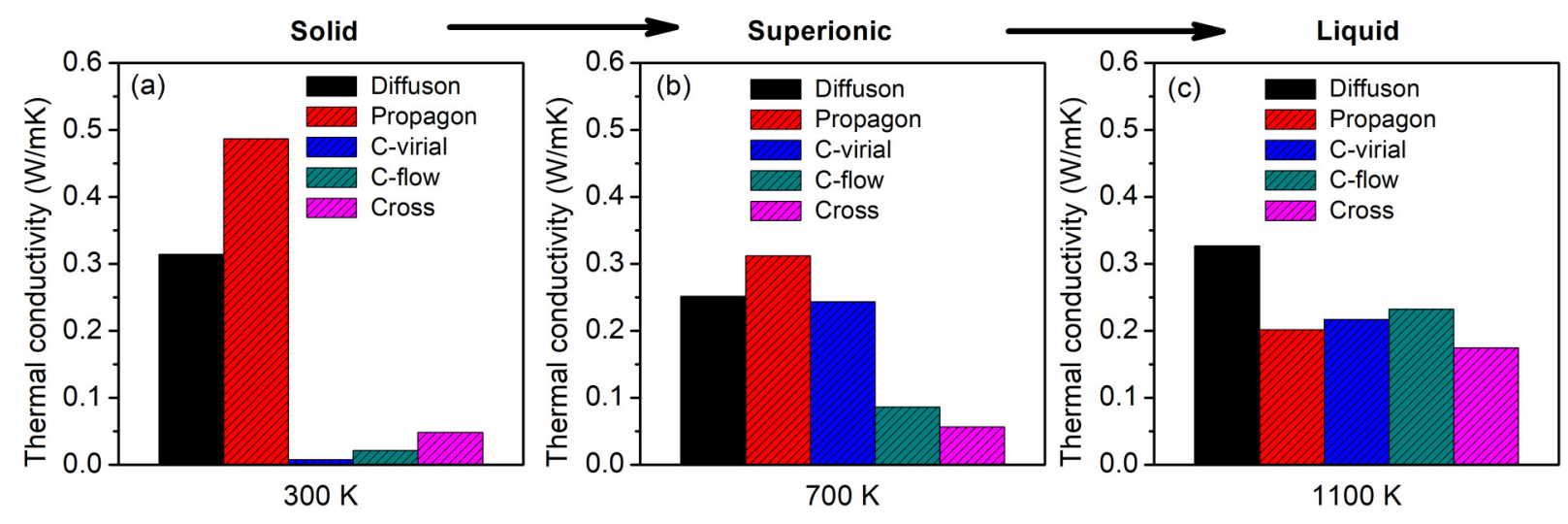

FIG. 10. Thermal conductivity contributed from each type of heat carrier in $\mathrm{Li}_{2} \mathrm{~S}$ in (a) the amorphous solid state (300 K), (b) the superionic state $(700 \mathrm{~K})$, and $(\mathrm{c})$ the liquid state $(1100 \mathrm{~K})$.

coupling between nonpropagating vibrations [6,7] or equivalently through the overlap of the vibrations' trajectories [14]. We then can calculate the corresponding thermal conductivities contributed from propagons and diffusons; details can be found in Sec. II C.

To facilitate the analysis of thermal conductivity contributions, the thermal conductivity accumulation function $\kappa_{\text {vibration }}\left(\Lambda_{\text {eff }}\right)$ of vibrational modes has been computed from the effective MFP $\Lambda_{\text {eff }}$ using [36]

$$
\kappa_{\text {vibration }}\left(\Lambda_{0}\right)=\sum_{\Lambda_{\text {eff }}<\Lambda_{0}} c_{v} v_{g} \Lambda_{\text {eff }},
$$

where $\Lambda_{0}$ represents the upper bound of $\Lambda_{\text {eff }}$ in the accumulative thermal conductivity function. Our results [Fig. 9(b)] show that the contribution to thermal conductivity from vibrational heat is $0.80 \mathrm{~W} / \mathrm{mK}$ at $300 \mathrm{~K}, 0.56 \mathrm{~W} / \mathrm{mK}$ at $700 \mathrm{~K}$, and $0.52 \mathrm{~W} / \mathrm{mK}$ at $1100 \mathrm{~K}$. It is not surprising that the thermal conductivity contributed from vibrations at $300 \mathrm{~K}$ is almost equal to the virial thermal conductivity because $\mathrm{Li}_{2} \mathrm{~S}$ is in its amorphous solid state at $300 \mathrm{~K}$ [Fig. 7(c)]. The main thermal transfer inside the system occurs via conduction, which is resulting from the virial terms in Eq. (1). Contrastingly, the system at $700 \mathrm{~K}(1100 \mathrm{~K})$ has a virial thermal conductivity of $0.79 \mathrm{~W} / \mathrm{mK}(0.83 \mathrm{~W} / \mathrm{mK})$ much larger than the one contributed by vibrations. As discussed above, $\mathrm{Li}$ ( $\mathrm{Li}$ and $\mathrm{S}$ ) ions do not vibrate around their equilibrium positions at $700 \mathrm{~K}$ $(1100 \mathrm{~K})$ and therefore the interatomic forces of the virial term not only contribute to conduction but also to convection.

\section{Heat carriers contributions}

Based on the above analysis, we now quantitatively characterize the contribution to heat exchange from propagons, diffusons, convection term due to interatomic forces, convection term due to the ions' flow, and, at last, the interaction between conduction and convection. Note that we estimate the contribution of the virial interatomic forces to convection $\kappa_{c \text {-virial }}$ via $\kappa_{c \text {-virial }}=\kappa_{\text {virial }}-\kappa_{\text {vibrations }}$.

At $300 \mathrm{~K}$ [Fig. 10(a) and Table II], diffusons and propagons appear as the two main heat carriers in amorphous $\mathrm{Li}_{2} \mathrm{~S}$, contributing to one-third and one-half of the total thermal conductivity, respectively. The virial thermal conductivity is almost equal to the summed thermal conductivity of diffusons and propagons. At $700 \mathrm{~K}$ [Fig. 10(b) and Table II], Li ions enter the liquid phase and therefore the convective motion among ions also carries one-third of the thermal energy. This convection thermal conductivity includes (i) the thermal energy carried by the ions' flow and (ii) the thermal energy transferred via virial interatomic interactions. The contribution from vibrations including propagons $(0.31 \mathrm{~W} / \mathrm{mK})$ and diffusons $(0.25 \mathrm{~W} / \mathrm{mK})$ becomes smaller because of the enhancement of the scattering among vibrations as well as of the one of the vibrations-ions' flow. When both $\mathrm{Li}$ and $\mathrm{S}$ ions flow at $1100 \mathrm{~K}$ [Fig. 10(c) and Table II], the convective motion of ions contributes to half of the total thermal energy exchange and the thermal conductivity resulting from the vibrations remains large with a value of $0.53 \mathrm{~W} / \mathrm{mK}$. We also find that the cross term contributed from the interaction between conduction and convection reaches $0.24 \mathrm{~W} / \mathrm{mK}$ and cannot be ignored in liquid $\mathrm{Li}_{2} \mathrm{~S}$.

\section{E. Transverse modes in the liquid phase}

We now explain why propagating modes appear in liquid $\mathrm{Li}_{2} \mathrm{~S}$ and why convection contributes via the virial heat flux in superionic and liquid $\mathrm{Li}_{2} \mathrm{~S}$. In a simple liquid, longitudinal acoustic modes are always observed in experiments [18-20] because the density fluctuations can occur due to stronger repulsive forces between atoms when they move closer to each other. The transverse modes are usually hardly detectable in liquids because the shear force in the system is very weak.

TABLE II. Contributions to thermal transfer of each heat carrier in $\mathrm{Li}_{2} \mathrm{~S}$ at $300 \mathrm{~K}, 700 \mathrm{~K}$, and $1100 \mathrm{~K}$.

\begin{tabular}{|c|c|c|c|c|c|}
\hline Temperature (K) & Diffusons (\%) & Propagons (\%) & C-virial (\%) & C-flow (\%) & Cross $(\%)$ \\
\hline 300 & 36.2 & 56.0 & 0.8 & 2.2 & 4.8 \\
\hline 700 & 27.5 & 34.0 & 24.3 & 8.6 & 5.6 \\
\hline
\end{tabular}



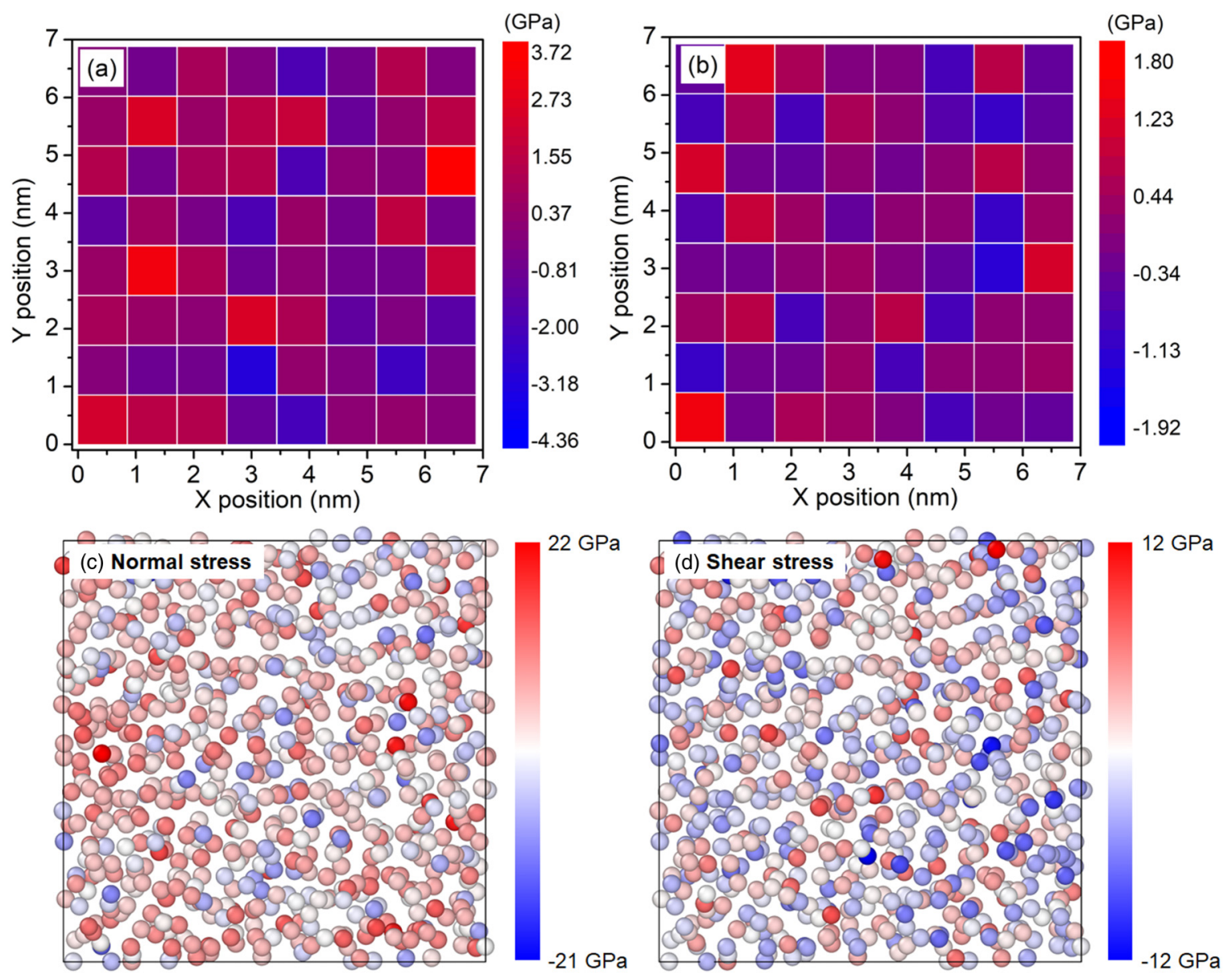

FIG. 11. (a) Normal stress and (b) shear stress spectral map of continuum amorphous liquid $\mathrm{Li}_{2} \mathrm{~S}$ at $1100 \mathrm{~K}$. (c) Atomistic normal stress $\sigma_{x x}$ and (d) atomistic shear stress $\sigma_{x y}$ spectral maps of liquid $\mathrm{Li}_{2} \mathrm{~S}$ at $1100 \mathrm{~K}$.

However, the situation changes when the vibrational effective MFP in a liquid approaches the nearest neighbor distance between atoms. In this case, a solidlike cage effect at the nanometer scale acts as a restoring force for transverse vibrational modes [18]. To further confirm this proposition, we first assume our system as a continuum medium with $8 \times 8 \times 8=$ 512 nodes [Figs. 11(a) and 11(b)]. Each node contains around 41 atoms. At long wavelengths, or equivalently for small wave vectors, the behavior of acoustic oscillations of node $i$ can be described by a continuum model, the displacement of which follows the equation of motion [37]

$$
\rho \frac{\partial^{2} u_{i}^{x}}{\partial t}=\sum_{\beta=x, y, z} \frac{\partial \sigma_{i}^{x \beta}}{\partial u_{i}^{\beta}}=\underbrace{\frac{\partial \sigma_{i}^{x x}}{\partial u_{i}^{x}}}_{\text {longitudinal related }}+\underbrace{\frac{\partial \sigma_{i}^{x y}}{\partial u_{i}^{y}}+\frac{\partial \sigma_{i}^{x z}}{\partial u_{i}^{z}}}_{\text {transverse related }},
$$

where $\sigma$ refers to the stress tensor of node $i, u_{i}^{\beta}$ is the node's displacement along the $\beta(\beta=x, y, z)$ direction, and $\rho$ is the density.
In simple liquids, longitudinal acoustic vibrational modes are easily observed by inelastic scattering experiments because the density fluctuations can occur due to a strong repulsive force between atoms when they approach each other as described by the first term in the right hand side of Eq. (18) [18], while the transverse vibrational modes are much more difficult to detect in liquids because the shear stress appearing in the last two terms of Eq. (18) is usually weak [18]. The stress distribution map of our amorphous liquid $\mathrm{Li}_{2} \mathrm{~S}$ [Figs. 11(a) and 11(b)] shows that the magnitude of the shear stress reaches a similar order compared to that of the normal stress, which indicates that the transverse vibrations modes should exist in amorphous liquid $\mathrm{Li}_{2} \mathrm{~S}$.

At the same time, the second term of the per-atom volume stress tensor $S$ used in LAMMPS [22] is calculated using

$$
S_{i}^{\alpha \beta}=\left\{\frac{1}{2} \sum_{i, j ; i \neq j}^{N} F_{i j}^{\alpha}(t) r_{i j}^{\beta}(t)+\sum_{i, j, k ; i \neq j \neq k}^{N} F_{i j k}^{\alpha}(t)\left[r_{i j}^{\beta}(t)+r_{i k}^{\beta}(t)\right]\right\},
$$


where $\alpha$ and $\beta$ ( $\alpha, \beta=x, y, z$ ) represent the directions. Then, the heat current in our calculations can also be written as

$$
\begin{aligned}
Q_{\text {viral }}^{x}(t) & =\frac{1}{V} V\left\{\sum_{i} S_{i}^{x x}(t) v_{i}^{x}(t)+\frac{1}{V} \sum_{i}^{N}\left[S_{i}^{y x}(t) v_{i}^{x}(t)+S_{i}^{z x}(t) v_{i}^{x}(t)\right]\right\} \\
& =\underbrace{V \sum_{i} \sigma_{i}^{x x}(t) v_{i}^{x}(t)}_{\text {longitudinal related }}+\underbrace{V \sum_{i}^{N}\left[\sigma_{i}^{y x}(t) v_{i}^{x}(t)+\sigma_{i}^{z x}(t) v_{i}^{x}(t)\right]}_{\text {transverse related }}
\end{aligned}
$$

in which $\sigma$ refers to the virial stress tensor of atom $i$ and is the stress plotted in Figs. 11(c) and 11(d). As we discussed above, the longitudinal vibrations are related to the normal stress [first term in Eq. (20)] and the transverse vibrations are resulting from the shear stress [second term in Eq. (20)]. As shown in our main text, the vibrational modes including both diffusons and propagons contribute more than two-thirds to the virial thermal conductivity calculated based on Eq. (20). We then infer that both the longitudinal and transverse vibrations are important for the thermal transport in liquid amorphous $\mathrm{Li}_{2} \mathrm{~S}$ when the first and second terms in Eq. (20) have similar magnitudes or, equivalently, the normal stress and the shear stress have similar magnitudes. Therefore, we may infer that the transverse modes in liquid $\mathrm{Li}_{2} \mathrm{~S}$ will also contribute largely to thermal transfer.

\section{CONCLUSIONS}

In conclusion, by performing reactive force field atomistic simulations and a dynamic structure factor analysis, we show the map of the quantitative contributions to heat exchange of each thermal excitation in $\mathrm{Li}_{2} \mathrm{~S}$ from the amorphous solid state to the superionic state and then to the liquid state. Our results reveal that the vibrations' scattering time is covering a wide interval ranging from a few femtoseconds to several picoseconds at $300 \mathrm{~K}$. As a result, both propagons and diffusons are the dominant heat carriers, while, for the superionic state, the vibrational thermal conductivity decreases from 0.81 $\mathrm{W} / \mathrm{mK}$ to $0.56 \mathrm{~W} / \mathrm{mK}$ due to the enhancement of vibrational scattering and the scattering between vibrations and the $\mathrm{Li}$ ions' flow. We also find that the thermal conductivity resulting from the convection heat transfer consists of (i) the heat carried by the ions' flow and (ii) the heat induced by atomic interactions via the cross correlation between the virial and the convection terms. For liquid $\mathrm{Li}_{2} \mathrm{~S}$ at $1100 \mathrm{~K}$, the convection conductivity due to the cross correlation is increased from a negligible value of $0.02 \mathrm{~W} / \mathrm{mK}$ at $300 \mathrm{~K}$ to $0.63 \mathrm{~W} / \mathrm{mK}$ at $1100 \mathrm{~K}$. The vibrational scattering time can still be as large as $\sim 1.5 \mathrm{ps}$. Thus conduction resulting from both propagons and diffusons decreases largely compared to its weight in solid $\mathrm{Li}_{2} \mathrm{~S}$, but remains quite substantial with a thermal conductivity value of $0.52 \mathrm{~W} / \mathrm{mK}$.

\section{ACKNOWLEDGMENTS}

Y.Z. thanks startup Fund (No. REC20EGR14, a/c-R9246) and HKUST-GZU joint research collaboration Fund (Grant No. GZU21EG06) from Hong Kong University of Science and Technology (HKUST).
[1] Y. Lan, A. J. Minnich, G. Chen, and Z. Ren, Adv. Funct. Mater. 20, 357 (2010).

[2] S. K. Bux, R. G. Blair, P. K. Gogna, H. Lee, G. Chen, M. S. Dresselhaus, R. B. Kaner, and J. P. Fleurial, Adv. Funct. Mater. 19, 2445 (2009).

[3] X. Huang, C. Zhu, Y. Lin, and G. Fang, Appl. Therm. Eng. 147, 841 (2019).

[4] D. G. Cahill, W. K. Ford, K. E. Goodson, G. D. Mahan, A. Majumdar, J. Humphrey, R. Merlin, S. R. Phillpot, W. K. Ford, and H. J. Maris, J. Appl. Phys. 93, 793 (2003).

[5] C. Kittel, Introduction to Solid State Physics (Wiley, New York, 1976).

[6] P. B. Allen and J. L. Feldman, Phys. Rev. B 48, 12581 (1993).

[7] J. Moon, B. Latour, and A. J. Minnich, Phys. Rev. B 97, 024201 (2018).

[8] J. M. Larkin and A. J. H. McGaughey, Phys. Rev. B 89, 144303 (2014).

[9] Y. Zhou and M. Hu, Phys. Rev. B 92, 195205 (2015).

[10] K. Sääskilahti, J. Oksanen, J. Tulkki, S. Volz, and A. J. H. Mcgaughey, AIP Adv. 6, 121904 (2016).
[11] Y. Zhou and M. Hu, Nano Lett. 16, 6178 (2016).

[12] D. G. Cahill, S. K. Watson, and R. O. Pohl, Phys. Rev. B 46, 6131 (1992).

[13] W. Lv and A. Henry, New J. Phys. 18, 013028 (2016).

[14] Y. Zhou, S. Xiong, X. Zhang, S. Volz, and M. Hu, Nat. Commun. 9, 4712 (2018).

[15] B. Wu, Y. Zhou, and M. Hu, J. Phys. Chem. Lett. 9, 5704 (2018).

[16] B. Li, H. Wang, Y. Kawakita, Q. Zhang, M. Feygenson, H. L. Yu, D. Wu, K. Ohara, T. Kikuchi, K. Shibata, T. Yamada, X. K. Ning, Y. Chen, J. Q. He, D. Vaknin, R. Q. Wu, K. Nakajima, and M. G. Kanatzidis, Nat. Mater. 17, 226 (2018).

[17] W. Qiu, L. Xi, P. Wei, X. Ke, J. Yang, and W. Zhang, Proc. Natl. Acad. Sci. USA 111, 15031 (2014).

[18] S. Hosokawa, M. Inui, Y. Kajihara, K. Matsuda, T. Ichitsubo, W. C. Pilgrim, H. Sinn, L. E. González, D. J. González, S. Tsutsui, and A. Q. R. Baron, Phys. Rev. Lett. 102, 105502 (2009).

[19] D. C. Elton and M. Fernández-Serra, Nat. Commun. 7, 10193 (2016). 
[20] A. D. B. Woods and R. A. Cowley, Phys. Rev. Lett. 24, 646 (1970).

[21] R. J. Hardy, Phys. Rev. 132, 168 (1963).

[22] S. Plimpton, J. Comput. Phys. 117, 1 (1995).

[23] D. Torii, T. Nakano, and T. Ohara, J. Chem. Phys. 128, 044504 (2008).

[24] P. Boone, H. Babaei, and C. E. Wilmer, J. Chem. Theory Comput. 15, 5579 (2019).

[25] R. Kubo, J. Phys. Soc. Jpn. 12, 570 (1957).

[26] M. M. Islam, A. Ostadhossein, O. Borodin, A. T. Yeates, W. W. Tipton, R. G. Hennig, N. Kumar, and A. C. T. Van Duin, Phys. Chem. Chem. Phys. 17, 3383 (2015).

[27] A. J. C. Ladd, B. Moran, and W. G. Hoover, Phys. Rev. B 34, 5058 (1986).

[28] S. Volz, J. B. Saulnier, M. Lallemand, B. Perrin, P. Depondt, and M. Mareschal, Phys. Rev. B 54, 340 (1996).
[29] S. Volz and G. Chen, Phys. Rev. B 61, 2651 (2000).

[30] A. J. H. McGaughey and M. Kaviany, Int. J. Heat Mass Transfer 47, 1799 (2004).

[31] P. Norouzzadeh, A. Nozariasbmarz, J. S. Krasinski, and D. Vashaee, J. Appl. Phys. 117, 214303 (2015).

[32] Y. M. Beltukov, C. Fusco, D. A. Parshin, and A. Tanguy, Phys. Rev. E 93, 023006 (2016).

[33] A. Kinaci, J. B. Haskins, and T. Çağin, J. Chem. Phys. 137, 014106 (2012).

[34] H. Shintani and H. Tanaka, Nat. Mater. 7, 870 (2008).

[35] E. L. Cussler, Diffusion: Mass Transfer in Fluid Systems (Cambridge University Press, Oxford, 2009).

[36] A. J. Minnich, J. A. Johnson, A. J. Schmidt, K. Esfarjani, M. S. Dresselhaus, K. A. Nelson, and G. Chen, Phys. Rev. Lett. 107, 095901 (2011).

[37] U. Böer and K. W. Pohl, Semiconductor Physics (Springer, New York, 2018). 